\title{
Diversidad genética de materiales nativos de aguacate guatemalteco a través del marcador molecular AFLP
}

\author{
Genetic diversity of native Guatemalan avocado through AFLP molecular marker \\ José A. Ruiz-Chután ${ }^{1,2 *}$, Julio E. Berdúo-Sandoval ${ }^{1}$, Marie Kalousová2, Bohdan Lojka², \\ Eloy Fernández², Jana Žiarovskáa ${ }^{3}$ Amílcar Sánchez-Pérez ${ }^{1}$ \\ ${ }^{1}$ Facultad de Agronomía, Universidad de San Carlos de Guatemala, Guatemala \\ ${ }^{2}$ Department of Crop Sciences and Agroforestry, Czech University of Life Sciences Prague, Czech Republic \\ ${ }^{3}$ Department of Genetics and Plant Breeding, Slovak University of Agriculture in Nitra, Slovak Republic
}

*Autor al que se dirige la correspondencia: josealejandro.ruiz@icloud.com

Recibido: 11 de abril 2019 / Revisión: 16 de enero 2020 / Aceptado: 09 de junio 2020

\section{Resumen}

— 1 aguacate es un cultivo de consumo a nivel mundial, y según teorías recientes, se sugiere a la región de la - Sierra Nevada, en California, como centro de origen y, a Guatemala, como uno de los principales centros de domesticación. Mediante caracterizaciones morfológicas se ha reportado una alta diversidad genética en el país, pero debido al comportamiento de polinización cruzada e hibridaciones interraciales, no se ha podido detallar el estado genético actual de la especie. Sin embargo, los marcadores moleculares son útiles para este tipo de estudios al enfocarse en las diferencias a nivel del ADN. Este estudio analizó la diversidad genética del aguacate nativo guatemalteco de siete poblaciones geográficas con el marcador molecular AFLP. Los datos de estructura poblacional mostraron un alto grado de diversidad a nivel de individuos $(\mathrm{Ht}=0.1933, \mathrm{Hw}=0.1872)$ y baja diferenciación entre poblaciones $(\mathrm{Hb}=0.0061)$. Los resultados sugieren una alta tasa de migración que influye directamente en el grado de mezcla genética de los materiales analizados. El bajo índice de estructura poblacional apunta a un alto flujo genético entre las poblaciones, por lo que la especie no presenta mayor riesgo ante la deriva genética, minimizándose el riesgo de pérdida de alelos por fijación. Se sugiere el resguardado del recurso fitogénetico total y no únicamente de materiales promisorios, evitando así el riesgo de erosión genética de la especie y garantizando la permanencia de la diversidad genética, la cual será la base de futuros programas de mejoramiento.

Palabras claves: Aguacate nativo, AFLP, estructura genética, domesticación, variabilidad genética

\begin{abstract}
$\mathrm{A}$ vocado is one of the most widely consumed crops worldwide and according to new theories, the Sierra Nevada region in California is suggested as the center of origin and Guatemala as one of the main domestication centers. Through morphological characterizations, a high genetic diversity has been reported in the country, but due to the behavior of cross pollination and interracial hybridizations, it has not been possible to detail the current genetic status of the species. Molecular markers are useful for this type of study by focusing on differences at DNA level. This study analyzed the genetic diversity of the native Guatemalan avocado from seven geographic populations with AFLP molecular marker. Population structure data showed a high degree of diversity at the individual level $(\mathrm{Ht}=0.1933, \mathrm{Hw}=0.1872)$ and low differentiation between populations $(\mathrm{Hb}=0.0061)$. The results suggest a high rate of migration that directly influences the degree of genetic mixing of the analyzed materials. The low index of population structure points to a high genetic flow between populations, so that the species does not present a greater risk due to genetic drift, minimizing the risk of loss of alleles due to fixation. The protection of the total genetic resource is suggested, and not only of promising materials, thus avoiding the risk of genetic erosion of the species and guaranteeing the permanence of genetic diversity, which will be the basis of future breeding programs.
\end{abstract}

Keywords: Native avocado, AFLP, genetic structure, domestication, genetic variability 


\section{Introducción}

El aguacate (Persea americana Mill.) pertenece a la familia Lauraceae, la cual incluye alrededor de 50 géneros, siendo considerada una de las familias de angiospermas más antiguas (Renner, 1999, 2004). El cultivo del aguacate, característico por acumular aceite en su pulpa en lugar de azúcares, es uno de los frutales de mayor importancia económica y cultural para la región de Mesoamérica. Se caracteriza por ser una especie perenne subtropical, aunque ha sido adaptada a diversos climas (Galindo et al., 2007; Galindo-Tovar \& Arzate-Fernández, 2010), por lo cual, su producción se ha extendido prácticamente a todo el continente americano, Asia, África, Medio Este y Europa (Guzmán et al., 2017). Según datos del Ministerio de Agricultura Ganadería y Alimentación (Maga, 2016) Guatemala tuvo una producción en el 2016 de 122,440.91 Tm de aguacate, los cuales fueron cosechados en un área de $11,572 \mathrm{ha}$, alcanzando un rendimiento de $10.58 \mathrm{Tm} / \mathrm{ha}$. Para el mismo año, tuvo una exportación de 4,028.24 Tm lo que representó un valor de US\$1,093,338 siendo El Salvador y Honduras los principales destinos del aguacate guatemalteco.

Respecto al centro de origen de la especie, han existido varias teorías desde que Popenoe (1935) propuso como centro de origen la región comprendida desde México hasta el norte de Sudamérica. Posteriormente, Williams (1977) menciona que el centro de origen del aguacate se localiza en las tierras altas del centro y centro-este de México, así como en las tierras altas de Guatemala. Storey y colaboradores (1986) proponen el área de Chiapas (sur de México) - Guatemala - Honduras como centro de origen basados en la identificación de posibles aguacates silvestres. Años después, Bergh (1992) menciona el área centro sur de México y Guatemala como el lugar donde pudo haberse originado la especie. El origen del aguacate se ha relacionado a las áreas donde actualmente existen poblaciones de gran diversidad o donde se han identificado individuos considerados como silvestres (Bergh, 1992; Storey et al., 1986). No obstante, Galindo-Tovar y Arzate-Fernández (2010) mencionan que no se han considerado los datos paleoclimáticos, ni la relación de la ubicación de los fósiles con la trayectoria del reemplazo biótico y las respuestas a las catástrofes ambientales, lo que según, dichos autores, ubicaría al centro de origen de la especie en una región mucho más al norte.

En el proceso de la reconstrucción de la historia del aguacate Scora y Bergh (1992) mencionan que el género Persea ya se encontraba en Norteamérica hace 56-35 millones de años, concordando con las condiciones de clima subtropical de la Sierra Nevada descritas para ese período (Millar, 1996) y con los fósiles de aguacate reportados por Schröeder (1968). Desde este escenario, es posible que al momento de la formación de la Sierra Nevada en el Cenozoico tardío (Liu \& Shen, 1998; Wakabayashi \& Sawyer, 2001) los primeros aguacates modernos se hayan originado por un proceso de adaptación en esta área y no más al sur en la zona de Chiapas - Guatemala - Honduras, en la cual no existían las condiciones climáticas favorables durante el Mioceno - Plioceno como describe Galindo-Tovar y Arzate-Fernández (2010). Estos autores también mencionan que, durante la última glaciación ocurrida en la Sierra Nevada, el clima cambió a seco y frío, por lo cual los aguacates se desplazaron hacia el sur quedando extintos en el área de origen.

En cuanto a la clasificación actual del aguacate, hasta el momento se reconocen tres razas hortícolas (mexicana, guatemalteca y antillana) (Bergh, 1992), las cuales están distribuidas en la región mesoamericana y, a partir de ellas, se han desarrollado la mayoría de variedades comerciales, aunque hay controversia en cuanto a su diferenciación debido a la alta variabilidad en la progenie (Bergh, 1992; Fiedler et al., 1998; Lavi et al., 2003). Previo a la llegada de los exploradores españoles, se asume que las razas fueron separadas con poca o ninguna movilidad debido a las condiciones topográficas, barreras climáticas y el gran tamaño de la semilla. Actualmente, las razas han sufrido cruzamiento en muchas regiones de América y numerosas colecciones muestran una clara introgresión racial. El hábito de floración y la polinización cruzada del aguacate, junto con su forma prolífica y un bajo conjunto de frutos, son otros factores que impiden la comprensión del linaje de los cultivares y razas actuales (Reyes-Alemán et al., 2016).

El tipo de evolución reticulada y la amplia complejidad genética, han dificultado el estudio de la diversidad y relaciones genéticas de esta especie. Actualmente, los estudios de diversidad genética se auxilian de marcadores morfológicos y moleculares, aunque la primera está sujeta a factores ambientales o fenológicos, siendo esto una fuerte limitante (Azofeifa-Delgado, 2006). Por otra parte, los marcadores moleculares son fenotípicamente neutros, presentan mayor segregación o polimorfismo que los morfológicos, pueden ser evaluados desde los primeros estados de desarrollo de las plántulas, son aplicables a cualquier material vegetal, 
no importa la época del año en que se realiza el análisis y están libres de los efectos epistáticos (Azofeifa-Delgado, 2006; Reyes-Alemán et al., 2017; Valadez-Moctezuma, Samah, \& Luna-Paez, 2014).

El desarrollo de los marcadores basados en el $\mathrm{ADN}$ ha tenido impacto en las investigaciones relacionadas al aguacate. Los marcadores basados en el polimorfismo de la longitud de los fragmentos de restricción (Restriction Fragment Lenght Polymorphism, RFLP) se utilizaron en estudios evolutivos, filogenéticos (Furnier et al., 1990) y genealógicos (Davis et al., 1998). Otros tipos de marcadores como los minisatélites fueron utilizados para estudios similares y con los mismos resultados en la identificación de las tres razas horticulturales ya descritas (Mhameed et al., 1997). Estudios similares fueron realizados empleando marcadores basados en el ADN polimórfico amplificado al azar (Random Amplified Polymorphic DNA, RAPD) (Fiedler et al., 1998; Álvarez Pecina et al., 2018). El desarrollo de microsatélites para esta especie (Ashworth et al., 2004; Gross-German \& Viruel, 2013) ha permitido la caracterización molecular de diversas colecciones, por ejemplo en Ghana (Acheampong et al., 2008), España (Alcaraz \& Hormaza, 2007) y México (Guzmán et al., 2017). Estudios a través de ISSR (Inter Simple Sequence Repeats) también han sido desarrollados con el objetivo de evaluar la variabilidad genética entre la raza mexicana (Cuiris-Pérez et al., 2009) y la diversidad presente entre el género Persea (Reyes-Alemán et al., 2017).

Asimismo, marcadores como los basados en el polimorfismo de la longitud de los fragmentos amplificados (Amplified Fragment Lenght Polymorphism, AFLP) también son útiles, a pesar de ser marcadores de tipo dominantes, debido a su alto poder discriminatorio. Este marcador molecular ha sido utilizando en el estudio de la diversidad genética del aguacate en Colombia (Cañas-Gutiérrez et al., 2015), México (Gutiérrez-Díez et al., 2009), Cuba (Nerdo et al., 2009; Ramírez et al., 2005); así como en la diversidad dentro de la raza mexicana (Cerda-Hurtado et al., 2015).

La investigación se planteó debido a la falta de una caracterización profunda a nivel molecular del recurso genético del aguacate guatemalteco, por ser este una fuente invaluable para programas de mejoramiento genético de la especie y por el potencial económico que este cultivo representa para el país. Para esto, se evaluó la diversidad genética de materiales nativos guatemaltecos en siete poblaciones, utilizando el marcador molecular AFLP.

\section{Materiales y métodos}

\section{Muestreo y colecta de material vegetal}

Basado en la información del atlas guatemalteco de parientes silvestres de plantas cultivadas (Azurdia et al., 2011) y tras el reconocimiento del área para la identificación de individuos nativos, se procedió a la colecta de 169 muestras. Estas fueron organizadas en siete poblaciones geográficas, abarcando cuatro regiones fisiográficas distintas. Las poblaciones fueron ubicadas en los departamentos de Sacatepéquez, Chimaltenango, Sololá, Totonicapán, Quiché, Huehuetenango, Alta Verapaz y Baja Verapaz; y en las regiones fisiográficas del Sur-Occidente, Nor-Occidente, Norte y Central, como se observa en la Figura 1. Debido a la naturaleza del estudio, de conocer la diversidad genética y la estructura poblacional de la especie, no se colectaron materiales que manifestaran indicios de mejoramiento o de pertenecer a colecciones de materiales seleccionados. El número de individuos por población fue variable debido a la accesibilidad y disponibilidad de materiales en cada una. Las muestras consistieron de cinco hojas frescas tomadas de la parte media de cada árbol, las cuales fueron almacenadas en condiciones de frío y trasladadas al laboratorio de biotecnología vegetal de la Facultad de Agronomía para realizar la extracción de ADN. Cada muestra fue identificada con número correlativo, nombre de la población y coordenadas geográficas.

\section{Extracción de ADN y protocolo de AFLP}

Para la extracción del ADN genómico se utilizó el kit comercial Gentra añadiendo algunas modificaciones para evitar la oxidación del material biológico. Se tomó una sección de cada hoja de aproximadamente 4 x $4 \mathrm{~mm}$ dentro de un tubo de $1.5 \mathrm{~mL}$ al cual se le agregaron 450 $\mu \mathrm{L}$ de Cell Lysis Solution (+ 1\% PVP, $+0.3 \%$ mercaptoetanol) y se procedió a macerar. Se agregaron 2.25 $\mu \mathrm{L}$ de proteinasa $\mathrm{K}(100 \mu \mathrm{g} / \mathrm{mL})$ y se dejó incubar por $3 \mathrm{~h}$ a $65^{\circ} \mathrm{C}$ realizando mezclas por inversión cada 30 min. Se agregaron $2.5 \mu \mathrm{L}$ de solución RNasa A (4 mg/ $\mathrm{mL}$ ) y se mezcló por inversión 25 veces dejando incubar nuevamente por $45 \mathrm{~min}$ a $37^{\circ} \mathrm{C}$. Posteriormente, se dejó enfriar a temperatura ambiente y se agregaron $150 \mu \mathrm{L}$ de solución para precipitación de proteínas, se mezcló 150 veces por inversión, se colocó por $20 \mathrm{~min}$ a $-20{ }^{\circ} \mathrm{C}$, se centrifugó a 14,000 rpm durante 4 min y 


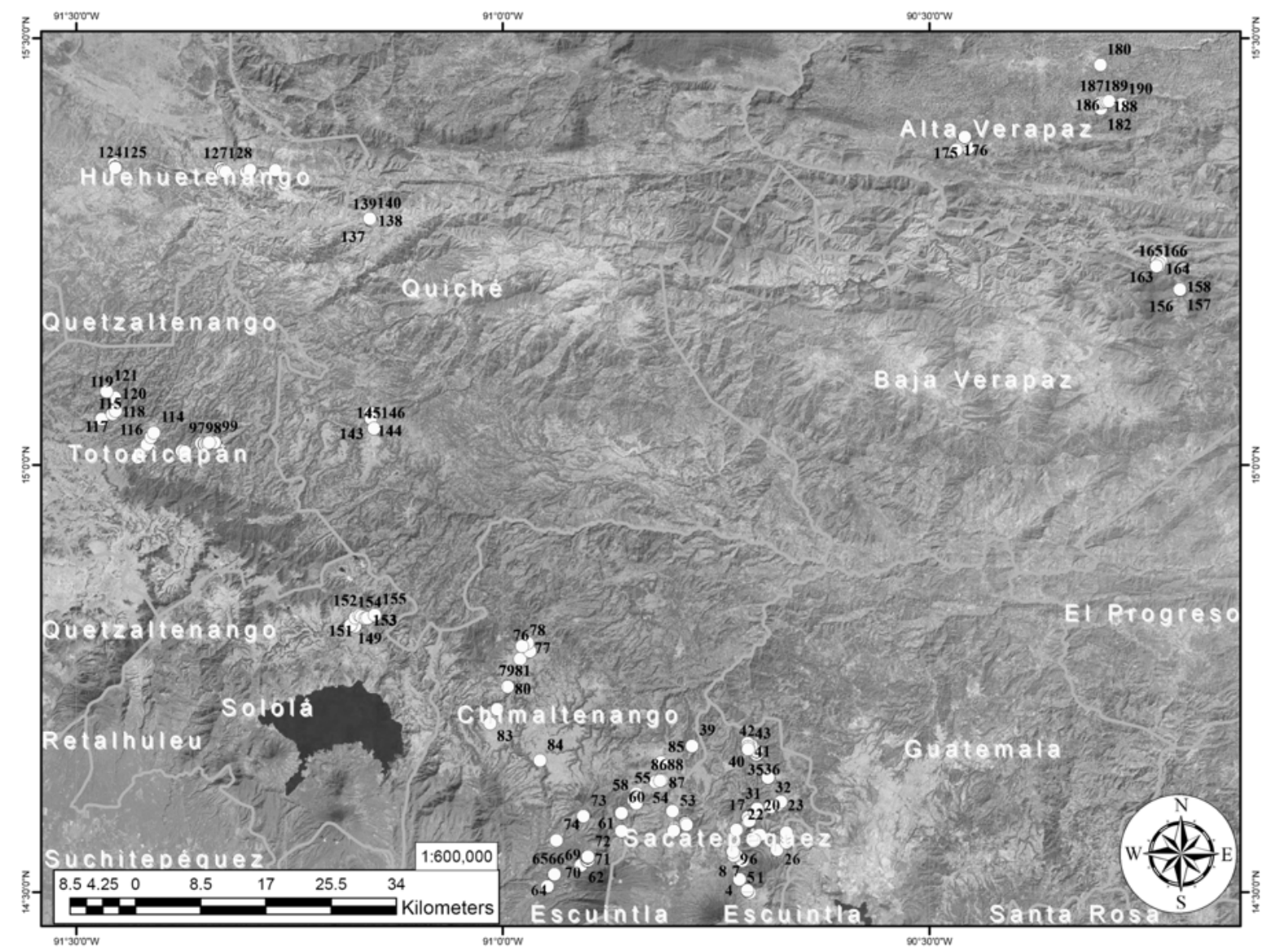

Figura 1. Sitios de colecta de materiales nativos de aguacate guatemalteco.

el sobrenadamente fue transferido a un nuevo tubo. Se agregaron $600 \mu \mathrm{L}$ de una solución fenol:cloroformo:alcohol isoamílico en una relación 25:24:1 se mezcló por inversión, se centrífugó nuevamente a 14,000 rpm por 10 min y el sobrenadante se transfirió a un nuevo tubo. Se agregaron $600 \mu \mathrm{L}$ de cloroformo (24): alcohol isoamílico (1) y se mezcló por inversión, se centrifugó por 10 min a $14,000 \mathrm{rpm}$ y el sobrenandante se transfirió a un nuevo tubo. Se agregaron $600 \mu \mathrm{L}$ de isopropanol y se mezcló por inversión 50 veces, se centrifugó por $3 \mathrm{~min}$ a 14,000 rpm, se descartó la fase líquida, se agregaron $500 \mu \mathrm{L}$ de etanol al $70 \%$, se mezcló por inversión para lavar la pastilla de $\mathrm{ADN}$, se centrifugó por $3 \mathrm{~min}$ a $14,000 \mathrm{rpm}$ invirtiendo el tubo para descartar el etanol y dejando secar por 10 a 15 min sobre papel absorbente. Para la hidratación del ADN se agregaron $50 \mu \mathrm{L}$ de la solución para hidratación de $\mathrm{ADN}$, se incubó a $65^{\circ} \mathrm{C}$ por $1 \mathrm{~h}$ y finalmente se almacenó a $-20^{\circ} \mathrm{C}$.
El marcador molecular AFLP (Vos et al., 1995) fue utilizado debido a la alta capacidad en la identificación de regiones polimórficas en estudios de diversidad genética de aguacate (Cañas-Gutiérrez et al., 2015; Cerda-Hurtado et al., 2015; Gutiérrez-Díez et al., 2009; Nerdo et al., 2009; Ramírez et al., 2005). El análisis se realizó con el Kit AFLP Análisis System I de Invitrogen ${ }^{\circledR}$ (Zabeau \& Vos, 1993). La metodología empleada fue la siguiente: se utilizó ADN genómico a una concentración de $25 \mathrm{ng} / \mu \mathrm{L}$, el cual fue digerido con las enzimas de restricción EcoR1/ Mse1 y se incubó por $2 \mathrm{~h}$ a $37^{\circ} \mathrm{C}$ y después a $70^{\circ} \mathrm{C}$ por $15 \mathrm{~min}$. La ligación de los adaptadores se llevó a cabo con los reactivos del kit y se incubó durante $2 \mathrm{~h}$ a $20^{\circ} \mathrm{C}$. Seguidamente se realizó una preamplificación con la reacción en cadena de la polimerasa; el programa empleado en el termociclador fue el siguiente: $94{ }^{\circ} \mathrm{C}$ por $30 \mathrm{~s}, 56{ }^{\circ} \mathrm{C}$ por $60 \mathrm{~s}, 72{ }^{\circ} \mathrm{C}$ por $60 \mathrm{~s}$, por 20 ciclos. Los productos 
amplificados se visualizaron en un gel de agarosa al $2 \%$. Se hizo una amplificación selectiva con los cebadores $\mathrm{M}-\mathrm{CAA}+\mathrm{E}-\mathrm{AAC}$ del kit los cuales produjeron mayor polimorfismo.

Los productos de la reacción en cadena de la polimerasa (PCR) se visualizaron en un gel de acrilamida al $5 \%$ para poder observar las bandas amplificadas. La tinción del gel se realizó con nitrato de plata mediante el siguiente procedimiento: inmersión en solución fijadora de ácido acético glacial $10 \% \mathrm{v} / \mathrm{v}$ durante $35 \mathrm{~min}$, agua destilada durante $20 \mathrm{~min}$, solución de tinción (nitrato de plata $0.15 \% \mathrm{p} / \mathrm{v}$, formaldehído $0.15 \% \mathrm{v} / \mathrm{v}$ ) por 40 min, agua destilada por $10 \mathrm{~s}$, solución de revelado de 5 a 6 min según la aparición de las bandas (carbonato de sodio $6 \% \mathrm{p} / \mathrm{v}$, formaldehído $0.3 \% \mathrm{v} / \mathrm{v}$, tiosulfato de sodio $5 \mathrm{ppm}$ ), solución fijadora para detener el proceso durante aproximadamente $5 \mathrm{~min}$, finalmente se lavó en agua destilada. Se dejó secar por 2 días y posteriormente se realizó la lectura del perfil de las bandas.

\section{Análisis de datos}

Con el perfil de bandas se generó una matriz binaria de presencia (1) ausencia (0) para cada uno de los loci amplificados, a partir de la cual se realizó el análisis de diversidad genética y estructura genética de poblaciones con el programa AFLP-Surv 1.0 (Vekemans et al., 2002). Para dicho análisis se estimaron el número de loci polimórficos (\# loc_P), porcentaje de loci polimórfico (PLP), heterocigosidad esperada bajo una proporción genotípica en equilibrio de Hardy-Weinberg $(\mathrm{Hj})$, diversidad genética promedio dentro de las poblaciones (Hw), diversidad genética promedio entre poblaciones $(\mathrm{Hb})$ diversidad genética total $(\mathrm{Ht})$, el índice estadístico de fijación de Wright (Fst) y la matriz de distancias genéticas con el índice de Nei. Para el análisis de inferencia sobre la estructura y división de las poblaciones analizadas a través del método bayesiano se empleó el programa Structure: V 2.3.4 (Pritchard et al., 2000)

Con el programa Paleontological Statistics Software Package for Education and Data Analysis Past3 (Ryan et al., 2001) se generó una matriz de distancias genéticas con el índice de Nei a partir del cual se desarrolló un análisis de conglomerados a través del método Unweighted Pair Group Method With Arithmetic Mean (UPGMA) (Sokal \& Michener, 1958) generando un dendrograma a nivel de individuos que fue exportado hacia el programa FigTree v1.4.3 (Rambaut, 2008) para generar el dendrograma de tipo circular. El análisis de conglomerados para generar el dendrograma a nivel de poblaciones se realizó con el programa R y el complemento "ape" (Paradis et al., 2004).

\section{Resultados}

Se analizaron 72 loci; el rango de loci polimórficos fue de 33 para la población de Sololá hasta 56 para la población de Chimaltenango, con un rango de porcentaje de loci polimórficos 45.8 a 77.8 para dichas poblaciones. El valor de heterocigosidad esperada por población estuvo en un rango de 0.13457 a 0.2652 siendo nuevamente la población de Chimaltenango la que presentó el valor más alto y la población de Sololá la que obtuvo el valor más bajo. El resumen de los datos puede observarse en la Tabla 1. El valor de diversidad genética total fue de 0.1933 , el valor de diversidad genética promedio dentro de las poblaciones fue de 0.1872 , la diversidad genética entre poblaciones fue de 0.0061 y finalmente, el valor de fijación de Wrigth fue de 0.0313 (Tabla 2.).

Con el análisis de conglomerados se generó un dendrograma (Figura 2), en el cual se observa una alta diversidad genética a nivel de individuos. Este mismo análisis demuestra que el grado de estructura o aislamiento genético entre las poblaciones es bajo. Este hecho se evidencia al existir una mezcla de individuos provenientes de las siete poblaciones analizadas. En este caso, la división en grupos claramente definidos no fue posible debido al alto grado de diversidad, cuantificado a través de los valores de distancias genéticas con el índice de Nei, entre los materiales analizados.

El análisis de conglomerados a nivel de poblaciones (Figura 3), muestra valores de distanciamiento genético muy bajos, indicando que las poblaciones comparten mucho de su material genético. Esta situación se observa claramente a través del índice $\mathrm{Hb}=0.0061 \mathrm{el}$ cual indica que, a pesar de existir una diferencia genética entre las poblaciones, esta es baja, por lo que no se observa un claro aislamiento genético entre las poblaciones. En el dendrograma las poblaciones se agruparon de la siguiente manera: Alta Verapaz y Baja Verapaz, Chimaltenango y Sacatepéquez, Quiché, Huehuetenango y Totonicapán; dejando como una población fuera de grupo a la proveniente de Sololá.

Esta situación también se reflejó con el análisis de comparación por pares de los valores de distancias genéticas a través del índice de Nei, en el cual se observa que los valores van en un rango de 0 entre las poblaciones de Alta Verapaz y Baja Verapaz, hasta un valor 
Tabla 1

Estructura genética de las poblaciones de aguacate nativo analizadas a través del marcador molecular AFLP

\begin{tabular}{|c|c|c|c|c|c|}
\hline Población & $\mathrm{N}$ & \# loci & \#loc_P & PLP & $\mathrm{Hj}$ \\
\hline AV & 18 & 72 & 39 & 54.2 & 0.17290 \\
\hline BV & 9 & 72 & 43 & 59.7 & 0.17198 \\
\hline Hue-Qui & 18 & 72 & 39 & 54.2 & 0.20120 \\
\hline Chimal & 10 & 72 & 56 & 77.8 & 0.26521 \\
\hline Sac-Chimal & 74 & 72 & 41 & 56.9 & 0.17932 \\
\hline Sol & 6 & 72 & 33 & 45.8 & 0.13457 \\
\hline Toto-Qui & 28 & 72 & 43 & 59.7 & 0.18520 \\
\hline
\end{tabular}

Nota . AV = Alta Verapaz, BV = Baja Verapaz, Hue-Qui = Huehuetenango - Quiché, Chimal = Chimaltenango, SacChimal $=$ Sacatepéquez - Chimaltenango, Sol $=$ Sololá, Toto-Qui $=$ Totonicapán - Quiché, N = número de individuos por población, \# loci = cantidad de loci analizados, \#loc_P = número de loci polimórficos, PLP = porcentaje de loci polimórficos, $\mathrm{Hj}$ = heterocigosidad esperada bajo una proporción genotípica en equilibrio de Hardy-Weinberg.

Tabla 2

Estructura genética de las poblaciones de aguacate nativo generada a través del marcador molecular AFLP

\begin{tabular}{lllll}
\hline $\mathrm{N}$ & $\mathrm{Ht}$ & $\mathrm{Hw}$ & $\mathrm{Hb}$ & Fst \\
\hline 7 & 0.1933 & 0.1872 & 0.0061 & 0.0313 \\
$\mathrm{~S} . \mathrm{E}$ & & 0.015082 & 0.001885 & 0.311799 \\
$\mathrm{Var}$ & 0.000227 & 0.000004 & 0.09 \\
\hline
\end{tabular}

Nota $. \mathrm{N}=$ número de poblaciones, $\mathrm{Ht}=$ diversidad genética total, $\mathrm{Hw}=$ diversidad genética promedio dentro de las poblaciones, $\mathrm{Hb}=$ diversidad genética entre las poblaciones, Fst = índice de fijación de Wright.

de 0.0281 entre las poblaciones de Chimaltenango y Sololá. Dichos valores pueden observarse en la Tabla 3.

La división de las poblaciones a través del análisis con el programa Structure mostró una mayor probabilidad en organizar las poblaciones en dos y cuatro grupos genéticos expresados a través del valor K (Figura 4). El grupo genético $(K=2)$ representado por la tonalidad más oscura, fue el dominante en la población de Sololá, mientras que, el grupo genético representado por la tonalidad clara, está ampliamente representado en las poblaciones de Chimaltenango y Huehuetenango-Quiché. En el resto de poblaciones, ningún grupo genético prevaleció, por lo que se asume una mezcla de dichos grupos. Cuando el valor $\mathrm{K}=4$ la población de Sololá fue dominada por el grupo genético representado en blanco, mientras en la población de Chimaltenango prevaleció el grupo genético representado por la tonalidad gris oscuro. Las demás poblaciones no mostraron una clara afinidad por ninguno de los grupos genéticos. 


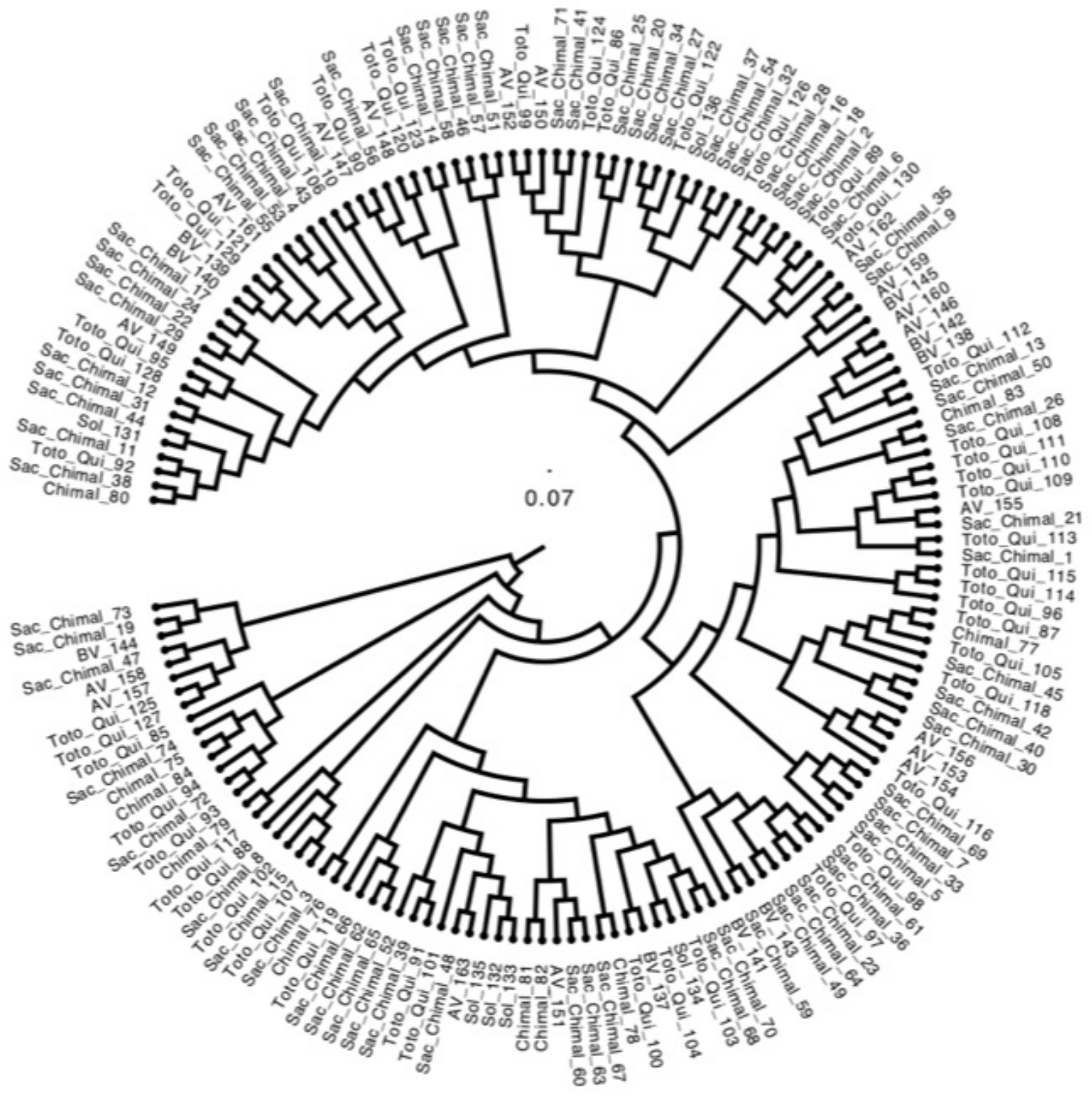

Figura 2. Dendrograma UPGMA generado a partir del marcador molecular amplified fragment length polymorphism (AFLP) utilizando las distancias genéticas de Nei que muestra la diversidad genética de aguacate nativo guatemalteco a nivel de individuos. Al número de cada individuo se antepuso la población de origen para facilitar su ubicación geográfica, AV = Alta Verapaz, BV = Baja Verapaz, Hue-Qui = Huehuetenango - Quiché, Chimal = Chimaltenango, Sac-Chimal $=$ Sacatepéquez - Chimaltenango, Sol $=$ Sololá, Toto-Qui = Totonicapán - Quiché. 


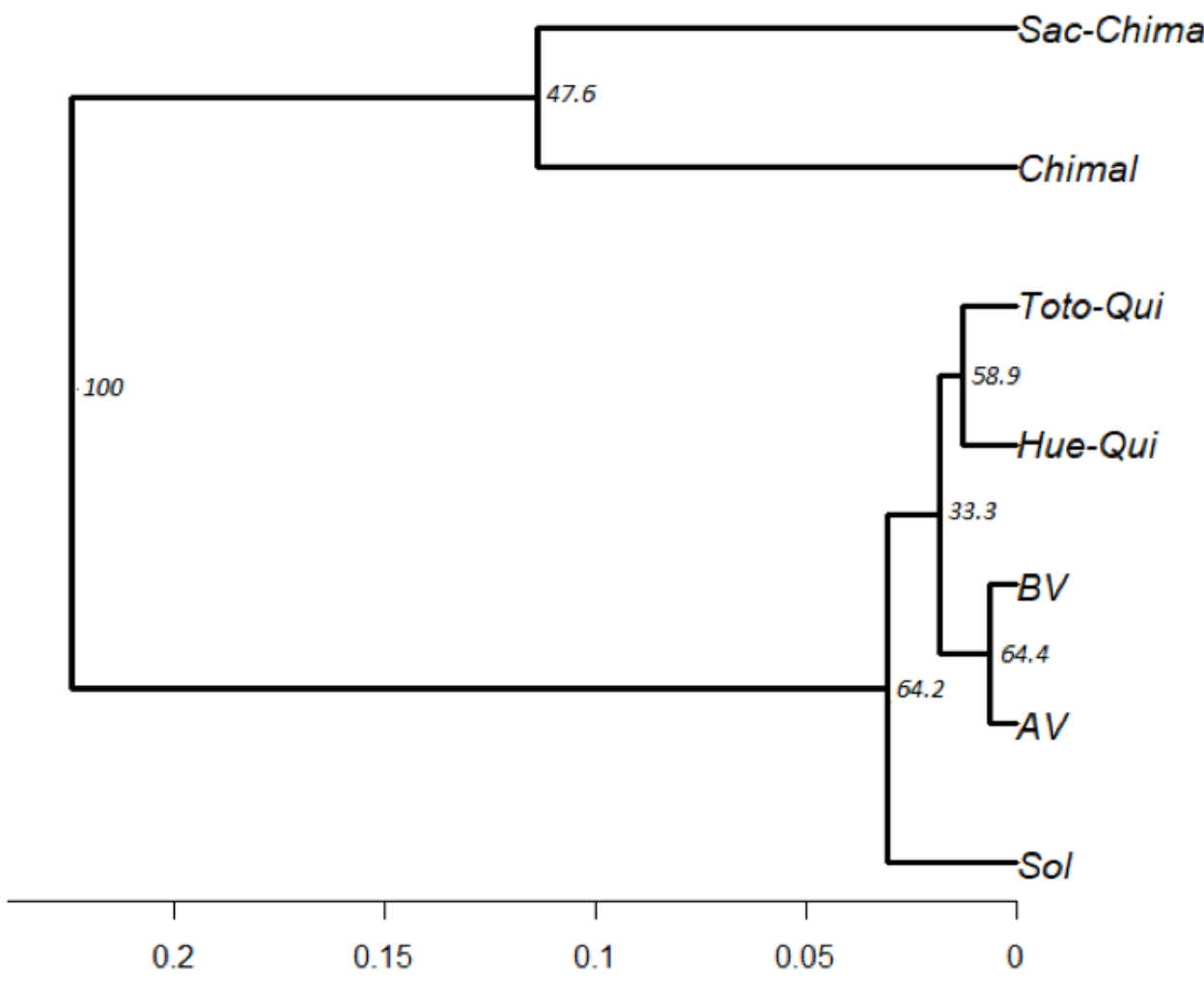

Figura 3. Dendrograma UPGMA generado a partir del marcador molecular amplified fragment length polymorphism (AFLP) utilizando las distancias genéticas de Nei que muestra la diversidad genética de aguacate nativo guatemalteco a nivel de poblaciones. Los números en cada uno de los clados muestran el valor de soporte estadístico con el método bootstraps después de 1,000 permutaciones.

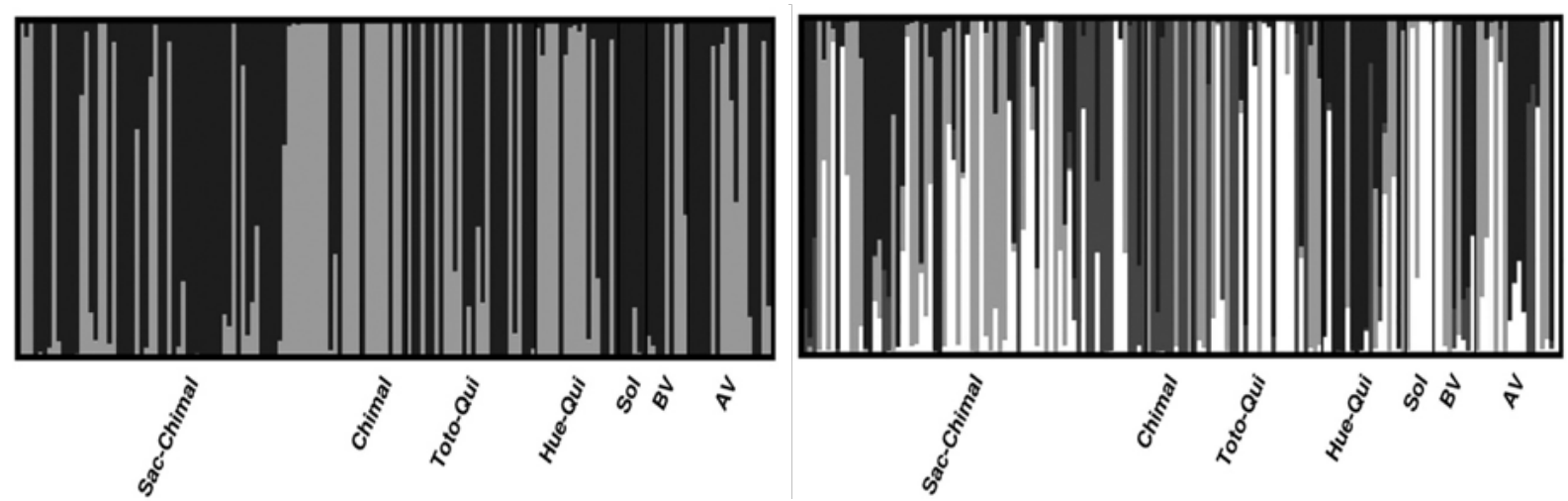

Figura 4. División de los materiales de aguacate nativo en dos $(\mathrm{K}=2)$ y cuatro $(\mathrm{K}=4)$ grupos genéticos inferidos por el programa Structure en el cual se observa el alto grado de mezcla genética entre las poblaciones. 
Tabla 3

Matriz de distancias genéticas generadas a partir del índice de Nei

\begin{tabular}{llllllll}
\hline Población & AV & BV & Hue-Qui & Chimal & Sac-Chimal & Sol & Toto-Qui \\
\hline AV & 0 & & & & & \\
BV & 0 & 0 & & & & \\
Hue-Qui & 0.0041 & 0.0079 & 0 & & & \\
Chimal & 0.0066 & 0.0098 & 0.0038 & 0 & & & \\
Sac-Chimal & 0 & 0.0014 & 0.0029 & 0.0056 & 0 & 0 & \\
Sol & 0.0086 & 0.0038 & 0.0268 & 0.0281 & 0.0169 & 0 & \\
Toto-Qui & 0 & 0 & 0.0058 & 0.0051 & 0.0004 & 0.0114 & 0 \\
\hline
\end{tabular}

Nota . AV = Alta Verapaz, BV = Baja Verapaz, Hue-Qui = Huehuetenango - Quiché, Chimal $=$ Chimaltenango, Sac-Chimal $=$ Sacatepéquez - Chimaltenango, Sol $=$ Sololá, Toto-Qui $=$ Totonicapán - Quiché .

\section{Discusión}

Para entender el comportamiento del aguacate nativo dentro del territorio guatemalteco, hace falta comprender que, en términos de genética de poblaciones, si las poblaciones presentan altos niveles de variación genética, esto indica que son muy grandes y/o que existe elevado flujo génico entre ellas. Por el contrario, si las poblaciones de una especie manifiestan marcadas diferencias entre sí, es posible que sean pequeñas y/o que exista poco flujo génico entre ellas (Eguiarte et al., 2013). Para el caso de este estudio, los índices de estructura poblacional apuntan a una alta conectividad entre las poblaciones, existiendo un elevado flujo génico, razón por la cual no se observa un claro aislamiento genético entre las poblaciones. Otro factor a considerar es que, las poblaciones con bajo flujo génico y de tamaño reducido, son susceptibles a sufrir los efectos de la deriva genética, lo que significa la pérdida de alelos por fijación (Labar \& Adami, 2017). Sin embargo, debido al flujo génico y la baja diferenciación entre las poblaciones analizadas, el riesgo de sufrir los efectos de la deriva genética, por parte del aguacate nativo, son menores.

Los esfuerzos para determinar el grado de diversidad genética en materiales de aguacate han llevado al uso del marcador molecular AFLP, mostrando ser de utilidad (Cerda-Hurtado et al., 2015). Además, otros marcadores moleculares de tipo dominantes como RAPD e ISSR (Fiedler et al., 1998; Reyes-Alemán et al., 2013) han permitido la caracterización molecular e identificación de diversidad genética de individuos dentro del género Persea y también dentro de la especie $P$. americana. Los resultados obtenidos por Cuiris-Pérez y colaboradores (2009) quienes evaluaron la diversidad genética dentro de la raza mexicana a través del marcador AFLP, obtuvieron un comportamiento muy similar a este estudio cuando se compara el nivel de organización a través del análisis de conglomerados mediante los dendrogramas generados.

La experiencia en la caracterización con el mismo marcador molecular (AFLP), de materiales nativos en el estado de Nuevo León, México (Gutiérrez-Díez et al., 2009), también mostró un alto grado de diversidad genética, aunque este estudio si logra un tipo de arreglo a través del dendrograma, debido a la previa caracterización a nivel de razas de las 42 accesiones analizadas. Sin embargo, debido al alcance del presente estudio, no se contó con la caracterización previa a nivel de razas, por lo que no fue posible establecer una relación como en el estudio antes mencionado, aunque si fue posible profundizar en los índices poblacionales.

Al examinar el comportamiento del aguacate nativo guatemalteco, se observa que el índice $\mathrm{Ht}(0.1933)$ 
que establece el grado de diversidad total y el índice $\mathrm{Hw}(0.1872)$ que mide el grado de diversidad genética entre el total individuos en cada población, refleja un alto grado de diferencia entre ellos. Esta situación es propia de una especie de polinización abierta, así como la condición de protoginia sincrónica que propicia la separación temporal de las funciones sexuales de la flor, favoreciendo así el entrecruzamiento (Bergh, 1974, 1992; Davenport, 1986). Este alto grado de variabilidad también responde al caso especial en su morfología floral denominado heterodicogamia, en el cual dos genotipos diferentes que presentan dicogamia sincronizada, tienen comportamiento de floración recíproca, lo cual significa que mientras las flores de uno de los individuos se encuentra en etapa femenina, las flores del otro se encuentran en la etapa masculina, favoreciendo así la polinización cruzada y aumento de variabilidad genética (Alcaraz \& Hormaza, 2011). Esto deja en claro que el aguacate posee un diseño que busca maximizar la polinización cruzada y de este modo incrementar la variabilidad genética y adaptación a la mayor cantidad de ambientes posibles, convirtiéndose en una especie altamente heterogénea.

Otro aspecto que ha ayudado a incrementar la diversidad del aguacate, es la ausencia de barreras de esterilidad entre las razas hortícolas de la especie, por lo cual es frecuente que se presenten hibridaciones interraciales de manera natural o inducida por el hombre (Gross-German \& Viruel, 2013). En cuanto a esto, las evidencias etnobotánicas (Storey et al., 1986; Williams, 1977) y los estudios genéticos (Ashworth \& Clegg, 2003; Furnier et al., 1990) sustentan el hecho de que las tres razas hortícolas permanecieron separadas durante su proceso de domesticación, pero tras la llegada de los españoles en el siglo XVI, estas entraron en contacto generándose las hibridaciones interraciales a causa del flujo genético originado por la acción humana. Esta situación ayuda a comprender la influencia de la interacción hombre - planta sobre la actual estructura poblacional del aguacate.

Cuando se examina el dendrograma a nivel de individuos (Figura 2) se observa que existe un alto grado de variabilidad entre estos, los cuales no pudieron ser ordenados en un arreglo de conglomerados que mantuviera relación con el origen geográfico de las poblaciones donde fueron colectados. Esta misma situación se presentó con el análisis de Structure (Figura 4) al no poderse establecer una total asignación de los individuos hacia los posibles grupos genéticos inferidos por el programa, observándose una mezcla de dichos grupos en la mayor parte de los individuos analizados. Este fenómeno, de acuerdo con Ashworth y Clegg (2003) es el resultado de la complejidad del estado híbrido de los aguacates, a lo que Bernal-Parra y colaboradores, 2014 agregan que, las especies alógamas, de amplia distribución geográfica y que están sujetas al intercambio de semillas, como es el caso del aguacate guatemalteco, incrementan considerablemente la variabilidad genética de los materiales.

El estudio de Galindo y colaboradores (2011), agrega que la antigua relación con el hombre y las diferentes condiciones de domesticación del aguacate han dado como resultado una gran diversidad de genotipos de afinidades genéticas muy complejas, como las encontradas en esta investigación, al igual que en el estudio de Cañas-Gutiérrez y colaboradores (2015). Por otra parte, al analizar el comportamiento del aguacate a nivel de poblaciones, es claro que el bajo valor del índice $\mathrm{Hb}$ (0.0061), el cual mide el grado de diferenciación genética entre poblaciones analizadas, indica que muchos de los loci identificados por el marcador molecular se encuentran presentes en todas las poblaciones. Dicha situación se interpreta como una alta similitud genética entre las poblaciones, situación que se ve respaldada por los bajos valores encontrados en la heterocigosidad esperada $(\mathrm{Hj})$. Esta situación indica que existe un flujo constante de materiales de aguacate a través de las poblaciones analizadas, lo cual genera dos condiciones, el incremento de la variabilidad genética entre todos los individuos por recombinación y un bajo nivel de estructura poblacional.

Este bajo nivel de estructura o aislamiento genético entre las poblaciones, puede ser claramente observado a través de la matriz de distancias genética (Tabla 3) ya que, para todos los casos de comparación, los valores obtenidos son muy cercanos a 0 , lo que se interpreta como una alta similitud genética entre las poblaciones. Esta misma situación también se identifica a través de los bajos valores de diferenciación genética en el dendrograma a nivel de poblaciones (Figura 3). Sin embargo, a pesar de existir un nivel muy bajo de diferenciación, este dendrograma si permite observar una relación positiva entre la cercanía genética y la cercanía geográfica, aunque los valores de bootstrap bajos indican que la estructura de los grupos identificados no están consolidados y pueden variar (Efron, 1979). Esta escasa estructura poblacional resulta, en gran medida, a la movilización de semillas entre los distintos departamentos del país, lo cual responde a la selección continua que realizan los productores nacionales en 
función de un idiotipo para satisfacer el mercado local o internacional (Ocampo, Urrea, Wyckhuys, \& Salazar, 2013). La comercialización de los frutos, a partir de los cuales se utilizan las semillas para el establecimiento de nuevas plantaciones, también es un factor que afecta la estructura genética.

El estudio de diversidad genética de aguacate realizado por Corona-Jácome, Galindo-Tovar, Lee-Espinosa y Landero-Torres (2016) que incluyó muestras tomadas en la región de Antigua Guatemala, encontró un alto valor de similitud genética entre dicha población y otra población ubicada en la localidad de Aquila, estado de Veracruz, México. Esta situación ayuda a explicar el comportamiento de la similitud genética de las poblaciones de aguacate guatemalteco, ya que los procesos de dispersión que sufrió la especie desde su posible origen en la Sierra Nevada (Galindo-Tovar \& Arzate-Fernández, 2010) han ayudado a la interacción entre las poblaciones, a pesar de existir grandes distancias geográficas entre ellas.

La situación genética de los materiales nativos en Guatemala merece especial atención, pues la estructura genética actual es resultado del efecto de la interacción humana a través del proceso de domesticación, el cual habría iniciado con la llega del hombre a Mesoamérica, hace aproximadamente 15,000 años (Goebel, Waters, \& O'Rourke, 2008). Desde entonces, las evidencias históricas, peleohistóricas y paleoecológicas indican que después del proceso de domesticación iniciado en México (Galindo-Tovar, Lee-Espinoza, Murgía-González, Leyva-Ovalle, \& Landero-Torres, 2013) los materiales de aguacate fueron trasladados hacia la zona de Yucatán, Guatemala y Belice, en donde la cultura maya estableció uno de los tres principales centros de domesticación del aguacate (Colunga-GarcíaMarín \& Zizumboo, 2004; Gama \& Gomez, 1992). A partir de ahí, las implicaciones religiosas, mitológicas, económicas y medicinales, que tuvo el aguacate en la región de Mesoamérica (Galindo-Tovar, Ogata-Aguilar, \& Arzate-Fernández, 2008), fueron fomentando el proceso de migración de materiales de aguacate fuera de sus poblaciones originales. Esta situación persiste en la actualidad y se ha convertido en el principal factor de incidencia en la diversidad y estructura genética de este valioso recurso.

Finalmente, la caracterización molecular con el marcador AFLP, permitió establecer que el germoplasma nativo de aguacate en Guatemala presenta un alto grado de variabilidad a nivel de individuos $(\mathrm{Ht}=$ $0.1933, \mathrm{Hw}=0.1872$ ) y bajo nivel de aislamiento entre poblaciones. Esta situación es atribuida al proceso de selección, al sistema de polinización cruzada, el intercambio de semillas y al proceso de domesticación, al que aún está sometido el aguacate; los cuales inciden en la variabilidad intraespecífica encontrada. Este estudio evidencia la riqueza genética del aguacate nativo guatemalteco y la importancia de su conservación. Sin embargo, para desarrollar una adecuada estrategia de conservación, es necesario tomar en cuenta que, la riqueza genética actual es producto del flujo génico entre las poblaciones. No obstante, este flujo se ve amenazado con la introducción, de forma extensiva, de variedades comerciales que desplazan a los materiales nativos, reduciendo su tamaño poblacional y dejándolos expuestos a los riesgos de deriva genética y endogamia. Por tanto, se sugiere mantener el tamaño y conectividad poblacional actual. También el resguardado del recurso fitogénetico total y no únicamente de materiales seleccionados por alguna característica de interés, evitando así el riesgo de erosión genética de la especie y garantizando la permanencia de la diversidad genética, la cual será la base de futuros programas de mejoramiento.

\section{Agradecimientos}

A la Dirección General de Investigación (Digi) por financiar la presente investigación a través de la partida presupuestaria 4.8.63.4.41

\section{Referencias}

Acheampong, A. K., Akromah, R., Ofori, F. A., Takrama, J. F., Saada, D., Bitton, I., \& Lavi, U. (2008). Genetic characterization of Ghanaian avocados using microsatellite markers. Journal of the American Society for Horticultural Science, 133(6), 801-809.

Alcaraz, M. L., \& Hormaza, J. I. (2007). Molecular characterization and genetic diversity in an avocado collection of cultivars and local Spanish genotypes using SSRs. Hereditas, 144, 244-253. https://doi.org/10.1111/j.2007.0018-0661.02019.x

Alcaraz, M. L., \& Hormaza, J. I. (2011). Influence of physical distance between cultivars on yield, outcrossing rate and selective fruit drop in avocado (Persea americana, Lauraceae). Annals of Applied Biology, 158(3), 354-361. https://doi. org/10.1111/j.1744-7348.2011.00469.x 
Álvarez, M.G., Pecina, V., Acosta, E., \& Almeyda. I. H. (2018). Evaluación molecular del aguacate criollo (Persea americana Mill) en Nuevo León, México. Revista Colombiana de Biotecnología, 20 (2), 3846. http://dx.doi.org/10.15446/rev.colomb.biote. v20n2.69551.

Ashworth, V. E. T. M., \& Clegg, M. T. (2003). Microsatellite markers in avocado (Persea americana Mill.): Genealogical relationships among cultivated avocado genotypes. Journal of Heredity, 94(5), 407-415. https://doi.org/10.1093/ jhered/esg076

Ashworth, V. E. T. M., Kobayashi, M. C., De la Cruz, M., \& Clegg, M. T. (2004). Microsatellite markers in avocado (Persea americana Mill.): Development of dinucleotide and trinucleotide markers. Scientia Horticulturae, 101, 255-267. https://doi.org/10.1016/j.scienta.2003.11.008

Azofeifa-Delgado, Á. (2006). Uso de marcadores moleculares en plantas; aplicaciones en frutales del trópico. Agronomía Mesoamericana, 17(2), 221-241.

Azurdia, C., Williams, K. A., Williams, D. E., Van Damme, V., Jarvis, A., \& Castaño, S. E. (2011). Atlas of Guatemalan crop wild relatives. Recuperado de http://www.ars.usda.gov/Services/ docs.html?docid $=22225$

Bergh, B. O. (1974). The remarkable avocado flower. California Avocado Society Yearbook, 57, 40-41.

Bergh, B. O. (1992). The origin, nature, and genetic improvement of the avocado. California Avocado Society Yearbook, 76, 61-75.

Bernal-Parra, N., Ocampo-Pérez, J., \& HernándezFernández, J. (2014). Caracterización y análisis de la variabilidad genética de la granadilla (Passiflora ligularis Juss.). Revista Brasileira de Fruticultura, 36(3), 586-597. https://doi. org/10.1590/0100-2945-251/13.

Cañas-Gutiérrez, G., Galindo-López, L., ArangoIsaza, R., \& Saldamando-Benjumea, C. (2015). Diversidad genética de cultivares de aguacate. Agronomía Mesoamericana, 26(1), 129-143. https://doi.org/10.15517/am.v26i1.16936

Cerda-Hurtado, I., Ojeda-Zacarías, M. Del C., IrachetaDonjuan, L., Martínez-De la Cerda, J., Torres-
Castillo, J. A., \& Gutiérrez-Díez, A. (2015). Variabilidad genética de cultivo in vitro de aguacate raza mexicana. Revista Mexicana de Ciencias Agrícolas, 6(1), 243-250.

Colunga-GaríaMarín, P., \& Zizumbo-Villareal, D. (2004). Domestication of plants in Maya lowlands. Economic Botany, 58, S101-110. https://doi.org/10.1663/0013-0001(2004)58[S10 1:DOPIML]2.0.CO;2

Corona-Jácome, E. C., Galindo-Tovar, M. E., LeeEspinosa, H. E., \& Landero-Torres, I. (2016). Diversidad genética del aguacate (Persea americana Mill.) en cuatro zonas de su área de dispersión natural. Agroproductividad, 9(6), 80-85.

Cuiris-Pérez, H., Guillén-Andrade, H., Pedraza-Santos, M., López-Medina, J., \& Vidales-Fernández, I. (2009). Genetic variability within mexican race avocado (Persea americana Mill.) germplasm collections determined by ISSRs. Revista Chapingo Serie Horticultura, 15(2), 169-175.

Davenport, T. (1986). Avocado flowering. Horticultural Reviews, 8, 257-289.

Davis, J., Henderson, D., Kobayashi, M., Clegg, M. T., Michael, T., \& Allen, P. C. K. (1998). Genealogical relationships among cultivated avocado as revealed through RFLP analyses. Journal of Heredity, 89(4), 319-323. https://doi. org/doi:10.1093/jhered/89.4.319

Eguiarte, L. E., Aguirre-Liguori, J. A., Jardón-Barbolla, L., Aguirre-Planter, E., \& Souza, V. (2013). Genómica de poblaciones: nada en evolución va a tener sentido si no es a la luz de la genómica, y nada en genómica tendrá sentido si no es a la luz de la evolución. Revista Especializada En Ciencias Químico-Biológicas, 16(1), 42-56. https://doi.org/10.1016/s1405-888x(13)72077-1

Efron, E. (1979). Bootstrap methods: Another look at the jackknife. Annals of Statistics, 7(1), 1-26.

Fiedler, J., Bufler, G., \& Bangerth, F. (1998). Genetic relationships of avocado (Persea americana Mill.) using RAPD markers. Euphytica, 101, 249255. https://doi.org/10.1023/a:1018321928400

Furnier, G. R., Cummings, M. P., \& Clegg, M. T. (1990). Evolution of the avocados as revealed by DNA restriction fragment variation. Journal of 
Heredity, 81(3), 183-188. https://doi.org10.1093/ oxfordjournals.jhered.a110963

Galindo-Tovar, M. E., \& Arzate-Fernández, A. (2010). Consideraciones sobre el origen y primera dispersión del aguacate Lauraceae. Cuadernos de Biodiversidad, 33, 11-15. http://dx.doi. org/10.14198/cdbio.2010.33.02

Galindo-Tovar, M., Arzate-Ferández, A., OgataAguilar, N., \& Landero-Torres, I. (2007). The avocado (Persea americana, Lauraceae) crop in mesoamerica: 10,000 years of history. Harvard Papers in Botany, 12(2), 325-334. https://doi. org/10.3100/1043-4534(2007)12[325:TAPALC ]2.0.CO;2

Galindo-Tovar, M. E, Milagro, P., Alejandre-Rosas, J., Leyva, O., Landero, I., Lee, H., \& Murguía, J. (2011). Relaciones genéticas del Aguacate (Persea americana Mill.) en siete municipios del centro de Veracruz, caracterizadas con microsatélites. Tropical and Subtropical Agroecosystems, 13, 339-346.

Galindo-Tovar, M. E., Lee-Espinoza, H. E., MurgíaGonzález, J., Leyva-Ovalle, O. R., \& LanderoTorres, I. (2013). Domesticación y distribución geográfica de Persea americana Mill. en la época precolombina. Revista de Geografia Agrícola, 5051, 65-70.

Galindo-Tovar, M. E., Ogata-Aguilar, N., \& ArzateFernández, A. (2008). Some aspects of avocado (Persea americana Mill.) diversity and domestication in Mesoamerica. Genetic Resources and Crop Evolution, 55, 441-450. https://doi.org/10.1007/s10722-007-9250-5

Gama, L., \& Gomez, P. (1992). An ethnoecological approach for the study of Persea: A case study in the Maya area. Proceedings II World Avocado Congress (pp. 11-17) Orange, California.

Gobel, T., Waters, M. R., \& O'Rourke, D. H. (2008). The late Pleistocene dispersal of modern humans in the Americas. Science, 319(5869), 1497-1502. https://doi.org/ 10.1126/science.1153569

Gross-German, E., \& Viruel, M. A. (2013). Molecular characterization of avocado germplasm with a new set of SSR and EST-SSR markers: Genetic diversity, population structure, and identification of race-specific markers in a group of cultivated genotypes. Tree Genetics \& Genomes, 9(2), 539555. https://doi.org/10.1007/s11295-012-0577-5 Gutiérrez-Díez, A., Martínez-de la Cerda, J., GarcíaZambrano, E. A., Iracheta-Donjuan, D., OcampoMorales, J. D., \& Cerda-Hurtado, I. M. (2009). Estudio de diversidad genética del aguacate nativo en Nuevo León, México. Revista Fitotecnia Mexicana, 32(1), 9-18.

Guzmán, L. F., Machida-Hirano, R., Borrayo, E., Cortés-Cruz, M., Espíndola-Baquera, M. de. C., \& Heredia, E. (2017). Genetic structure and selection of a core collection for long term conservation of avocado in Mexico. Frontiers in Plant Science, 8, 1-10. https://doi.org/10.3389/ fpls.2017.00243

Labar, T., \& Adami, C. (2017). Evolution of drift robustness in small populations. Nature Communications, 8(1), 1-12. https://doi. org/10.1038/s41467-017-01003-7

Lavi, U., Sa'ada, D., Regev, I., \& Lahav, E. (2003). Avocado genetics and breeding - present and future. World Avocado Congress V, 42, 134-135.

Liu, M., \& Shen, Y. (1998). Sierra Nevada uplift: A ductile link to mantle upwelling under the basin and range province. Geology, 26(4), 299-302. https://doi.org/10.1130/00917613(1998)026<0299:SNUADL >2.3.CO;2

Ministerio de Agricultura, Ganadería y Alimentación. (2016). El Agro en cifras, 2016. Guatemala: Autor Mhameed, S., Sharon, D., Kaufman, D., Lahav, E., Hillel, J., \& Lavi, U. (1997). Genetic relationships within avocado (Persea americana Mill) cultivars and between Persea species. Theoretical Applied Genetics, 94, 279-286. https://doi.org/10.1007/ s001220050411

Millar, C. I. (1996). Tertiary vegetation history. Sierra Nevada Ecosystem Project, Final report to Congress, Volume II, Assessments and Scientific Basis for Management Options, Centers for water and Wildland Resources, Report No. 37, University of California, Davis California

Nerdo, N., Medina, R., Luis, J., Lorenzo, F., Arbelo, O. C., Fiallo, R. F., ... Rohde, W. (2009). Agro-morphologic traits, isoenzyme and DNA 
markers for estimating the polymorphism levels, discriminating capacity and informativeness in avocado. Revista CENIC Ciencias Biologicas, 40(1), 63-74.

Ocampo, J., Urrea, R., Wyckhuys, K., \& Salazar, M. (2013). Aprovechamiento de la variabilidad genética del maracuyá (Passiflora edulis f. flavicarpa Degener) como base para un programa de fitomejoramiento en Colombia. Acta Agronomica, 62(4), 352-360.

Paradis, E., Claude, J., \& Strimmer, K. (2004). APE: Analyses of phylogenetics and evolution in $\mathrm{R}$ language. Bioinformatics, 20(2), 289-290. https:// doi.org/10.1093/bioinformatics/btg412

Popenoe, W. (1935). Origin of the cultivated races of avocados. California Avocado Association Yearbook, 20, 184-194.

Pritchard, J. K., Stephens, M., \& Donnelly, P. (2000). Inference of population structure using multilocus genotype data. Genetics, 155(2), 945-959. https:// doi.org/10.1111/j.1471-8286.2007.01758.x

Rambaut, A. (2008). FigTree v1.4.3: Tree figure drawing tool.

Ramírez, I. M., Fuentes, J. L., Rodríguez, N. N., Coto, O., Cueto, J., Becker, D., \& Rohde, W. (2005). Diversity analysis of Cuban avocado varieties based on agro-morphological traits and DNA polymorphisms. Journal of Genetics and Breeding, 59(3-4), 241-252. https://doi. org/10.1051/0004-6361/201117847

Renner, S. S. (1999). Circumscription and phylogeny of the Laurales: evidence from molecular and morphological data. American Journal of Botany, 86(9), 1301-1315.

Renner, S. S. (2004). Variation in diversity among Laurales, early Cretaceous to present. Biologiske Skrifter, 55, 441-458.

Reyes-Alemán, J. C., Serrano-Hernández, M., Mejía-Carranza, J., Vázquez-García, L. M., Urbina-Sánchez, E., Valadez-Moctezuma, E., \& Espíndola-Barquera, M. (2017). A genetic diversity study of Persea. Acta Horticulturae, (1203), 147-154. https://doi.org/10.17660/ ActaHortic.2018.1203.22
Reyes-Alemán, J., Valadez-Moctezuma, E., \& Barrientos-Priego, A. (2016). Assessment of genetic relationship in Persea spp by traditional molecular markers. Genetic and Molecular Research, 15(2), 1-11. https://doi.org/10.4238/ gmr.15027359

Reyes-Alemán, J. C., Valadez-Moctezuma, E., SimutaVelázco, L., Barrientos-Priego, A., \& GallegosVázquez, C. (2013). Distinción de especies del género Persea mediante RAPD e ISSR de ADN. Revista Mexicana de Ciencias Agrícolas, 4(4), 517-529.

Ryan, P. D., Hammer, Ø., \& Harper, D. A. (2001). Past: Paleontological statistics software package for education and data analysis. Palaeontologia Electronica, 4(1), 5-7. https://doi.org/10.1016/j. bcp.2008.05.025

Schröeder, C. A. (1968). Prehistoric avocados in California. California Avocado Association Yearbook, 52, 29-34.

Scora, R., \& Bergh, B. (1992). Origin of the taxonomic relationships within the genus Persea. En Proceedings II World Avocado Congress (pp. 505-514). California.

Sokal, R. R., \& Michener, C. D. (1958). A statistical methods for evaluating relationships. University of Kansas Science Bulletin, 38, 1409-1448.

Storey, W. B., Bergh, B., \& Zentmyer, G. A. (1986). The origin, indigenous range and dissemination of the avocado. California Avocado Society Yearbook, 70, 127-133.

Valadez-Moctezuma, E., Samah, S., \& Luna-Paez, A. (2014). Genetic diversity of Opuntia spp. varieties assessed by classical marker tools (RAPD and ISSR). Plant Systematics and Evolution, 301(2), 737-747. https://doi.org/10.1007/s00606-0141112-y

Vekemans, X., Beauwens, M., Lemaire, M., \& RuizRoldán, I. (2002). Data from amplified fragment length polymorphism (AFLP) markers show indication of size homoplasy and of a relationship between degree of homoplasy and fragment size X. Molecular Ecology, 11, 139-151. https://doi. org/10.1046/j.0962-1083.2001.01415.X 
Vos, P., Hogers, R., Bleeker, M., Reijans, M., van De Lee, T., Hornes, ... Zabeau, M. (1995). AFLP: A new technique for DNA fingerprinting. Nucleic Acids Research, 23(21), 4407- 4414. https://doi. org/10.1093/nar/23.21.4407

Wakabayashi, J., \& Sawyer, T. L. (2001). Stream incision, tectonics, uplift, and evolution of topography of the Sierra Nevada, California. The Journal of Geology, 109, 539-562. https:// doi.org/1010.1086/321962
Williams, L. O. (1977). The Avocados, a synopsis of the genus Persea, subg. Persea. Economic Botany, 31(3), 315-320. https://doi.org/10.1007/ BF02866883

Zabeau, M., \& Vos, P. (1993). Selective restriction fragment amplification: A general method for DNA fingerprinting (European patent application no. 92402629.7), European Patent Office. Recuperado de https://patentimages.storage. googleapis.com/49/1f/be/97e98216ef4ca3/ EP0534858A1.pdf 